\title{
Thermal spot velocimetry inside a boundary layer in water
}

\author{
by E. Koroteeva*, I. Znamenskaya*, P. Ryazanov* and A. Shagiyanova* \\ * Lomonosov Moscow State University, 119991, Leninskie Gory, 1, Moscow, Russia, koroteva@physics.msu.ru
}

\begin{abstract}
We propose a new method for quantitative visualization of the flow-velocity fields within a boundary layer of a non-isothermal fluid using seedless velocimetry. The method is based on the high-speed thermal imaging of the dynamics of temperature inhomogeneities through an infrared transparent wall. When the temperature inhomogeneities act as a passive flow tracer, the processing of the thermal-image sequences using a cross-correlation algorithm yields the velocity fields in a near-wall flow. Here, we study the near-wall flow formed in the region of interaction between a submerged impinging water jet and a flat plate. The obtained flow velocities range up to $1 \mathrm{~m} / \mathrm{s}$, and the limits of the laminar-turbulent transition inside the boundary layer are determined.
\end{abstract}

\section{Introduction}

The development of different flow visualization techniques is important for understanding the fluid flow structure, as well as from the viewpoint of validation of numerical models and algorithms. Despite the advances in temporal and spatial resolution of available measurement devices (both contact ad noncontact), the boundary layer region for near-wall flows remains hard to resolve. At the same time the boundary region - where the flow interacts with the solid - is of particular interest in the area of heat and mass transfer [1, 2].

The current achievements in infrared technology allowed for new approaches to flow visualization. Recently, we have proposed an infrared-based method for the quantitative analysis of turbulent characteristics of near-wall liquid flows $[3,4]$. The method implies high-speed imaging of turbulent thermal fluctuations from a near-wall boundary layer through solid windows transparent to mid-wave infrared radiation. Here, we propose and examine a new technique for a seedless flow velocimetry inside liquid boundary layers - Thermal Spot Velocimetry (TSV) - which is a combination of high-speed infrared thermography and cross-correlation post processing algorithms.

In this work, the TSV method is applied to study the non-isothermal flow resulting from the interaction between a submerged impinging water jet and a solid surface.

\section{Experimental setup and image processing}

In experiments, a water jet of a temperature $T_{\text {jet }}=10-50{ }^{\circ} \mathrm{C}$ is exhausted from a round nozzle of diameter $\mathrm{D}$ at a set flow rate. The nozzle is placed in a glass tank filled with water at a temperature of $\mathrm{T}_{0}=5-60^{\circ} \mathrm{C}$ orthogonally to a target plate at a distance $\mathrm{H}$. The target plate is a circular window made of calcium fluoride, which has a high transmittance in the mid-wave infrared spectral range (around 95\%). The experiments are conducted for a range of nozzle diameters, $D=1-4 \mathrm{~mm}$, nozzle-to-plate distances, $\mathrm{H} / \mathrm{D}=1-6$, and jet velocities, $\mathrm{v}_{\mathrm{jet}}=0.5-5 \mathrm{~m} / \mathrm{s}$. The thermal imaging is performed using a FLIR SC7700 MWIR camera $(3.7-4.8 \mu \mathrm{m})$ at frame rates up to $400 \mathrm{~Hz}$.

The proposed post-processing method is based on following the dynamics of "thermal spots" in the infrared images. We define a "thermal spot" as an element of a non-isothermal turbulent flow with the smallest possible size that emits infrared radiation of specific intensity. In turbulent flows, with the characteristic time scales much smaller than those for heat transfer, the temperature of the thermal spots remains constant. As a result, the spatial shift of each spot between two successive images defines the flow direction and velocity. Thus, by applying the PIV algorithm to the pairs of thermal images, we can obtain the velocity vector fields within the near-wall layer of the flow.

\section{Results}

Fig. 1 shows a mean velocity field for an impinging jet with parameters: $D=1.5 \mathrm{~mm}, \mathrm{H} / \mathrm{D}=2, \mathrm{v}_{\text {jet }}=5 \mathrm{~m} / \mathrm{s}$. The vector map is obtained by averaging the results of post-processing of 100 consecutive thermal-image pairs (recorded at a frame rate of $295 \mathrm{~Hz}$ ). The distribution of absolute velocity values are presented in grayscale, and the vectors indicate the direction of the velocities in the surface plane.

The analysis of the velocity vector fields in the wall-jet flow of a submerged impinging water jet in a wide range of flow parameters (nozzle diameters, nozzle-to-plate distances, jet Reynolds numbers) revealed the similarity of the flow structure: the velocity field appears axisymmetric and has an unresolved region, few nozzle diameters in size. Further downstream from the unresolved region the near-wall flow velocity increases to its maximum, and then decreases with increasing the radial distance from the stagnation point. The unresolved region indicates a zone where the thermal spots cannot be captured within the infrared emitting water layer, i.e. where there is no turbulent mixing of a warm jet with cold ambient fluid. 
Taken the strong absorption of MWIR radiation by liquid water [5], the emitting layer thickness does not exceed $0.4 \mathrm{~mm}$. We also conducted stand-alone experiments to assess the effective thickness of the water emitting layer for a range of initial jet conditions and to analyze the TSV results [6]. Our measurements showed that, for the conditions of the present experiment, the obtained velocity fields show the average flow velocities in a 0.2-mm-thick layer near the impinging surface. Thus, the appearance of the velocity vectors in the TSV images can be interpreted as the onset of intensive turbulent mixing within this thin boundary layer.

The variation of the temperature contrast between the jet and the surrounding fluid $\left(T_{j e t}-T_{0}\right)$ gave insight into the temperature sensitivity and limitations of the proposed method.

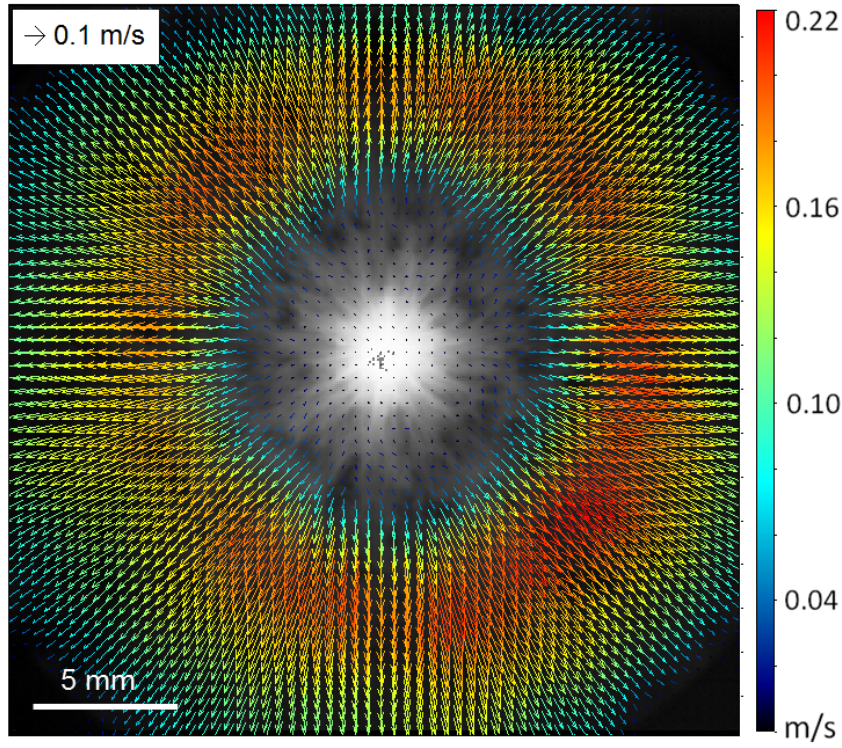

Fig. 1. Mean TSV velocity field for a submerged impinging jet with parameters: $D=1.5 \mathrm{~mm}, H / D=2, v_{j e t}=5$ $\mathrm{m} / \mathrm{s}$.

\section{Conclusion}

The proposed Thermal Spot Velocimetry method opens up new possibilities for studying the quantitative dynamic characteristics of turbulent flows near a solid wall. The described investigation algorithm can be used both for experimental determination of the turbulent-flow structure and the boundaries of the laminar-turbulent transition in a fluid and for validation and testing of numerical models describing the motion of a fluid near the surface.

The work is supported by Russian Science Foundation (Grant number 19-79-00162).

\section{REFERENCES}

[1] Lienhard, J. H. (V), Liu, X., Gabour, L. A. "Splattering and heat transfer during impingement of a turbulent liquid jet" J. Heat Transfer, Trans. ASME. 114, 1992.

[2] Carlomagno GM, laniro A "Thermo-fluid-dynamics of submerged jets impinging at short nozzle-to-plate distance: a review", Exp. Thermal Fluid Sci, vol. 58, pp. 15-35, 2014.

[3] I. A. Znamenskaya, E. Yu Koroteeva, A. M. Novinskaya, and N. N. Sysoev "Spectral peculiarities of turbulent pulsations of submerged water jets", Technical Physics Letters, Vol. 42(7), pp. 686-688, 2016.

[4] Znamenskaya I., Koroteeva E., Shagiyanova A. "Thermographic analysis of turbulent non-isothermal water boundary layer", Journal of Flow Visualization and Image Processing, Vol. 26, no. 1, pp. 49-56, 2019.

[5] L. Kou, D. Labrie, P. Chylek, "Refractive indices of water and ice in the 065- to 25- $\mu \mathrm{m}$ spectral range," Applied Optics, vol. 32, no. 19, pp. 3531, 1993.

[6] A.M. Shagiyanova etal "Analysis of the Visualization Region in Near-Wall Fluid Layer by High-Speed Infrared Thermography", Moscow University Physics Bulletin, Vol. 75, No. 2, pp. 143-147, 2020. 